WS9-A03

Increasing Transmitter Current and Reducing
Ambient Noise Levels - What Are the Limitations?

R. Mittet* (EMGS), J.P. Morten (EMGS) \& H.R. Jensen (EMGS)

\title{
SUMMARY
}

The marine controlled-source electromagnetic method (CSEM) can detect subsurface hydrocarbon reservoirs because they represent resistors in a conductive medium, i.e. brine-saturated rocks. The limitations on the applicability of the technology are given by target burial depth, lateral extent, and the net pay thickness. Improvements to the acquisition instrumentation can extend the applicability and increase the resolution. However, several factors affect the accuracy of the measured data. To achieve a significant improvement it is important to understand the experimental error contribution from each hardware component in relation to the target effect on the data. We present error propagation analysis for CSEM acquisition, which reveals feasible limitations for target detection. Further, we show how equipment can be optimally improved to extend applicability of the technology. 


\section{Theory and example}

If we want to detect an object in the subsurface with the marine CSEM method, then the emitted electromagnetic field must reach that object with significant amplitude. The field amplitude decays during propagation because of attenuation in the conductive formation. Moreover, we require that the field response must also come back to the receiver with amplitude that is above the measurement uncertainty. Qualitatively, there are two different sources for measurement uncertainty. When the field response is weak, random noise in the environment or noise generated by the receiver electronics can significantly distort the data. This gives rise to a signal-to-noise limitation on the measurement. An additional source of uncertainty is due to fluctuations associated with the emission and detection of the CSEM signal. For example, random amplitude errors from a fluctuating source antenna current will corrupt even a strong target response.

The measurement uncertainty is estimated by the application of error propagation analysis as described in Mittet and Morten (2012). The uncertainty in the inline electric field can be expressed,

$$
\delta E_{x}(x)=\sqrt{\gamma(x)^{2}\left|E_{x}^{O b s}(x)\right|^{2}+\Delta N^{2}},
$$

where $x$ is the source-receiver offset, $\gamma(x)$ parameterizes the relative-error contributions to the uncertainty that scale with the amplitude of the observed field, $\left|E_{x}^{O b s}(x)\right|$, and the ambient noise term $\Delta N$ contains contributions to the uncertainty that are independent of the amplitude of the observed field. The scale factor $\gamma(x)$ has a strong offset dependence for near offsets. For offsets above approximately $2 \mathrm{~km}$ this scale factor can be assumed constant with offset, at least for intermediate and deep water surveying. Sensitivity to a deeply buried target requires that the target response exceeds the measurement uncertainty. We therefore require that the amplitude of the scattered field, $\Delta E_{x}(x)$, from the resistive anomaly that we want to identify, is larger than the field uncertainty, $\delta E_{x}(x)$. Thus the signal-to-uncertainty ratio, $\Psi(x)=\Delta E_{x}(x) / \delta E_{x}(x)$, must be larger than unity over some substantial offset interval if the observed data are sensitive to a resistive anomaly. The offset interval where we demand $\Psi(x)>1$ is determined by imaging resolution criteria.

Consider a case of low sensitivity. The signal-to-uncertainty ratio can potentially be increased by increasing source strength to transmit an increased current. The amplitude of the scattered field will be proportional to the amplitude of the transmitted current. But the uncertainty will also increase. If the measurement uncertainty were dominated by the relative errors then the uncertainty $\gamma(x)\left|E_{x}^{O b s}(x)\right|$ will also be proportional to the amplitude of the transmitted current, and the sensitivity measure $\Psi(x)$ remains unchanged. In the described case it becomes important to reduce the relative error contribution to the uncertainty in order to take full advantage of the increased transmitter current. The error propagation analysis is a tool to understand how different components of the equipment used in a CSEM survey contribute to both $\gamma$ and $\Delta N$. The relative-error contribution to the uncertainty will typically depend on source and navigation accuracy, and instrument calibration accuracy. The ambient noise contribution depends on the receiver self-noise, but also on MT noise, swell noise, and motion noise induced by local water currents. The interplay between $\gamma$ and $\Delta N$ is shown in Figure 1. 


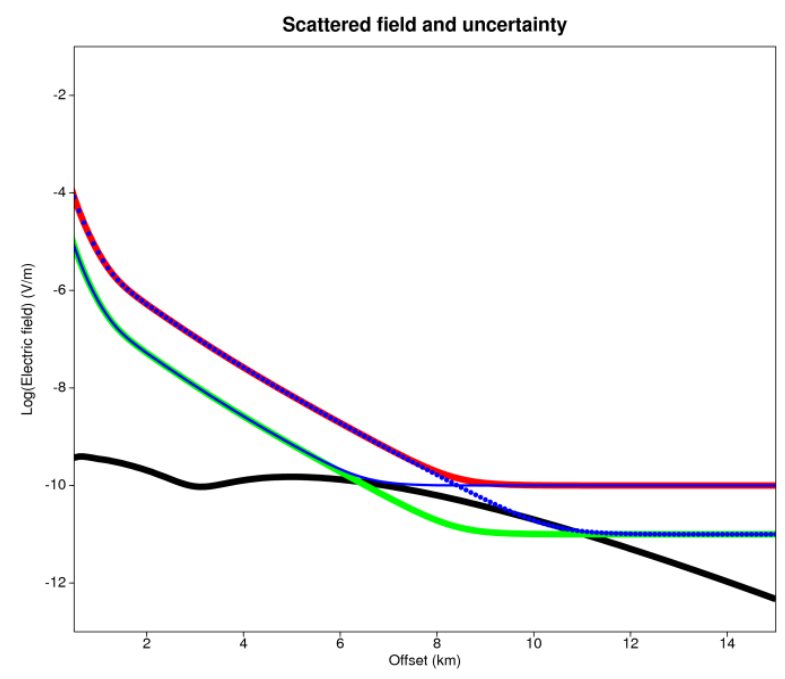

\section{Amsterdam |'14}

Figure 1 The black line is the scattered field $\Delta E_{x}(x)$. The colored lines are different realizations of the uncertainty $\delta E_{x}(x)$. The red line is for $\gamma=0.05$ and $\Delta N=10^{-10} \mathrm{~V} / \mathrm{m}$. The dotted blue line is for $\gamma=0.05$ and $\Delta N=10^{-11} \mathrm{~V} / \mathrm{m}$. The solid blue line is for $\gamma=0.005$ and $\Delta N=10^{-10} \mathrm{~V} / \mathrm{m}$, and the green line is for $\gamma=0.005$ and $\Delta N=10^{-11} \mathrm{~V} / \mathrm{m}$.

Before proceeding we need to specify the scattered field $\Delta E_{x}(x)$. The case we analyze here is the field scattered from a thin resistive layer. We use,

$$
\Delta E_{x}(x)=\left|E_{x}^{o b s}(x)-E_{x}^{B c k}(x)\right|,
$$

where the background field $E_{x}^{B c k}$ is calculated in a resistivity model with a $2 \mathrm{~km}$ water layer above a half space with $2 \mathrm{Ohm}-\mathrm{m}$. The observed field is calculated for the same model, except that there is a resistive target layer $4.4 \mathrm{~km}$ below the seabed. This layer is $50 \mathrm{~m}$ thick and has a resistivity of 50 Ohm-m. The frequency is $0.5 \mathrm{~Hz}$. The transmitted current has amplitude $10 \mathrm{kA}$.

In Figure 1 the scattered field, $\Delta E_{x}(x)$, is shown with the black line. The colored lines are different realizations of the field uncertainty, $\delta E_{x}(x)$, with parameters as explained in the figure caption. If the survey equipment is characterized by $\gamma=0.05$ and $\Delta N=10^{-10} \mathrm{~V} / \mathrm{m}$ (red line), we observe that the field uncertainty is always larger than the amplitude of the scattered field and a reasonable conclusion is that there is no sensitivity to the target layer. What if the ambient noise level is reduced with an order of magnitude? This is shown by the dotted blue line $\left(\gamma=0.05\right.$ and $\left.\Delta N=10^{-11} \mathrm{~V} / \mathrm{m}\right)$, and it is clear that the uncertainty remains equal to or above the amplitude of the scattered field for all offsets. Again it is reasonable to conclude that there is a negligible sensitivity to the target layer. The alternative situation where the ambient noise level is $\Delta N=10^{-10} \mathrm{~V} / \mathrm{m}$ but the relative-error term is reduced to $\gamma=0.005$ (solid blue line) give a similar conclusion as above; no sensitivity to the target layer. Only when both ambient noise and relative-error contributions to the uncertainty are reduced sufficiently (green curve) do we achieve sensitivity to the target layer for this case.

It is also worth to notice that a reduced transmitter current amplitude of $1 \mathrm{kA}$, but with $\gamma=0.005$ and $\Delta N=10^{-11} \mathrm{~V} / \mathrm{m}$ will give data that are insensitive to the target layer. In summary, it is therefore essential to reduce the ambient noise level in order to increase the maximum depth of sensitivity. Likewise, it also is essential to increase the transmitter current amplitude in order to increase the maximum depth of sensitivity, but increasing the transmitter current amplitude may have little effect unless the relative-error contribution to the uncertainty is reduced to a sufficient level.

\section{Conclusions}

In this presentation we will elaborate the error propagation analysis described above. We will show how it can be used as a tool to make detailed predictions of how improvements to various components of the instrumentation impact the ability of the data to detect a resistive target. Acknowledgements: The authors wish to thank EMGS for permission to publish these results.

\section{References}

Mittet, R. and J. P. Morten [2012] Detection and imaging sensitivity of the marine CSEM method. Geophysics, 77 (6), E411-E425. 